\title{
Correction to: Synergistic effect of cytokinins and auxins enables mass clonal multiplication of drumstick tree (Moringa oleifera Lam.): a wonder
}

\author{
Swati Gupta $^{1} \cdot$ Sumita Kachhwaha ${ }^{2} \cdot$ Shanker Lal Kothari $^{3} \cdot$ Rohit Jain $^{1}$ \\ Published online: 19 February 2021 \\ (C) The Society for In Vitro Biology 2020
}

Correction to: In Vitro In Vitro Cellular \& Developmental Biology - Plant https://doi.org/10.1007/s11627-020-10065-0

The word "tree" was inadvertently deleted from end of the title of this article as published.

The corrected title of the article is as follows:

Synergistic effect of cytokinins and auxins enables clonal mass multiplication of drumstick (Moringa oleifera Lam.): a wonder tree

The online version of the original article can be found at https://doi.org/ 10.1007/s11627-020-10065-0

\section{Rohit Jain}

rohit.jain@jaipur.manipal.edu

1 Department of Biosciences, Manipal University Jaipur, Jaipur, India

2 Department of Botany, University of Rajasthan, Jaipur, India

3 Amity University Rajasthan, Jaipur, India 